\title{
EL ABURRIMIENTO EN EL TRABAJO: APORTES RECIENTES DE LA PSICOLOGÍA LABORAL
}

\section{Boredom at work: Work Psychology's recent contributions}

\author{
Arturo Solf Zárate* \\ Universidad Femenina del Sagrado Corazón
}

\begin{abstract}
Resumen
El presente trabajo está relacionado a la experiencia de aburrimiento del personal en los centros de trabajo, así como sus consecuencias. Se resalta los aportes de la investigación empírica, la cual recientemente se ha incrementado frente a la evidencia de un continuo aumento de dicho estado. El texto incluye aspectos como: definición, características, medición, análisis del proceso. Seguidamente se encuentran algunas sugerencias para la realización de futuras investigaciones sobre el tema. Finalmente se presentan orientaciones prácticas para su prevención o disminución.
\end{abstract}

Palabras clave: Aburrimiento laboral, roles del psicólogo organizacional, bienestar psicológico, gestión de recursos humanos

\begin{abstract}
This paper is related to the experience of boredom of personnel in workplaces, as well as its consequences. The contributions of empirical research, which has recently increased against the evidence of a continuous rise of that state, are emphasized

The text includes aspects such as: definition, characteristics, measurement, and process analysis. Then, there are some suggestions to carry out future researches on the subject. Finally,practical guidelines are presented for its prevention or diminution.
\end{abstract}

Key words: Work boredom, organizational psychologist's roles, psychological well-being, human resources management.

\footnotetext{
* Psicólogo. Docente en las Universidades: UNIFÉ, USIL. arturo.solf@gmail.com
} 


\section{INTRODUCCIÓN}

Ultimamente se observa mayor interés de los especialistas por indagar y estudiar el aburrimiento en el trabajo, tal es así, que en el año 2015 han publicado en revistas de psicología más de 326 artículos al respecto (van Tilburg \& Igou, 2017). Este interés ha sido fomentado principalmente por los cambios en las organizaciones actuales, en las que "...observamos personas que ocupan puestos de trabajo que no les retan llevándolos a experimentar desinterés y poca estimulación." (Martínez \& Rodríguez- Montalbán, 2017, p.8), agregándose a esto la continua modernización tecnológica, que asigna al trabajador un rol menos protagónico (Cummings, Gao, \& Thornburg, 2016).

El aburrimiento como estado afectivo negativo merece ser prevenido o disminuido, al estar vinculado a problemas de bienestar psicológico y al desempeño inadecuado de los trabajadores (Balzer, Jex \& Geimer, 2017).

En el presente trabajo se incluyen aspectos relacionados a la definición del aburrimiento, sus características identificados por los investigadores, su medición, y un análisis desde la perspectiva de proceso. Luego se proponen algunas sugerencias a fin de propiciar su investigación, y finalmente se incluyen posibles acciones prácticas para prevenir o disminuir dicha emoción.

Para su elaboración se consideró en primer lugar a los estudiantes de psicología interesados en especializarse en el área de Psicología Organizacional, así como a los psicólogos que estén apoyando profesionalmente a los centros de trabajo. Otro sector, que quizás le puede interesar, incluye a consultores y supervisores habituados a utilizar los aportes psicológicos para una gestión eficaz de los recursos humanos.

\section{DEFINICIÓN, CARACTERÍSTICAS Y TIPOS DE ABURRIMIENTO}

El concepto de aburrimiento es complejo al incluir dimensiones cognitivas, afectivas, conductuales, y fisiológicas. Hasta la fecha no se ha formulado una definición aceptada por la mayoría de los especialistas, pero la más utilizada es la propuesta de Fisher (1993) quien llegó a definir el aburrimiento como “....un estado transitorio desagradable, en que el individuo siente una falta de interés y dificultad para concentrarse en la actividad actual" (p. 396; citado por Skowronski, 2012).

Para tener un mayor conocimiento sobre las características del aburrimiento, a continuación, mencionamos los aportes de tres investigaciones al respecto.

Eastwood, Frischen, Fenske, y Smilek (2012) analizaron las definiciones de aburrimiento según las perspectivas: psicodinámicas, existenciales, de activación, y cognitivas, concluyendo que todas ellas presentan como descriptores comunes del aburrimiento, el "estado aversivo de desear algo, pero incapaz de comprometerse en una actividad satisfactoria" (p.483). Esta conclusión constituye su propuesta para definir al aburrimiento. Además, sostienen que el elemento central del aburrimiento radica en el control de la atención de parte del sujeto. Específicamente, proponen al "... aburrimiento ser definido en términos de atención. Esto es, el estado aversivo que ocurre cuando (...)" (p.483):

- No logramos otorgar la atención debida a determinada información interna (p.ej., pensamientos, sentimientos) o externa (p.ej., recursos medioambientales) necesaria para realizar una actividad.

- Es difícil concentrarse y actuar para una participación eficaz según las circunstancias

- "Atribuimos la causa de nuestro estado aversivo al medioambiente" (p. 483) (p.ej., "esta tarea es aburrida").

Paralelo al marco de atención presente en el aburrimiento, Eastwood et al., (2012) identifican algunos componentes experienciales, lo que vivencia el sujeto durante dicho estado:

- Conciencia de la dificultad para concentrarse. Quizás por el esfuerzo adicional para realizar una tarea que no lo atrae, o al "divagar la mente" sobre contenidos más gratificantes.

- Sentir que los estímulos externos le exigen poco o demasiado para sus capacidades y experticia 
- Presencia adicional de otros sentimientos, como tristeza, ansiedad, vacuidad, cólera.

- Conciencia de estar presionado por factores externos para permanecer en una determinada situación y no poder hacer algo que desearía ("agencia desordenada").

- Percibir que el tiempo avanza muy lento. Sensación de estar en un presente sin final. "Esta experiencia es a veces asociada con sentimientos de 'desperdiciar el tiempo' antes que perseguir actividades productivas" (Eastwood et al., 2012, p. 489). Las personas pueden ser más sensibles al paso del tiempo al no concentrarse en una actividad atractiva, y más bien orientan su atención a ciertas claves internas (p.ej., hora de ingerir sus alimentos) o externas (p.ej., un reloj en el ambiente) más vinculadas con el paso del tiempo.

Es bueno indicar que lo anterior mencionado se limita a la descripción que Eastwood et al. (2012) realizan sobre del aburrimiento según sus componentes mentales y experienciales, sin considerar las causas de dicho estado que podrían ser: personalidad propensa al aburrimiento, baja motivación, lesión cerebral, problemas de sueño, psicopatología, etc.

La segunda investigación corresponde a los psicólogos van Tilburg e Igou (2017) quienes efectuaron tres estudios para esclarecer en qué difiere el aburrimiento de otras emociones negativas. Utilizaron muestras de trabajadores y estudiantes norteamericanos. Para la comparación consideraron las siguientes emociones: tristeza, cólera, frustración, depresión, culpa, vergüenza, temor, disgusto, pena, y desilusión. Después del análisis de los datos identificaron que el aburrimiento era muy diferente de las demás por su falta de significatividad y baja atención del sujeto para sus labores. Además, identificaron otras diferencias menos intensas que "...sugieren que el aburrimiento posee una valencia negativa moderada, es de baja activación, y se asocia con un desafío percibido bajo (...)" (p.309). Estos resultados les permitieron afirmar que el aburrimiento "...es considerablemente distinto de otras emociones negativas y su estudio científico específico por lo tanto está justificado.” (p. 320)

Anteriormente van Tilburg e Igou (2012) en base a cuatro estudios, encontraron que el aburrimiento se diferenciaba de las demás emociones negativas por la falta de significado y el bajo desafío que percibían las personas en relación a sus tareas o situación. Además, indicaron que, si bien estas dos dimensiones permitían distinguirla de las demás emociones negativas, no implicaba ser lo único característico del aburrimiento, ya que se debe considerar como una "configuración de experiencias" de modo que "...la gente aburrida siente inquietud y no desafío, piensan que la situación no sirve para un propósito, y desean comprometerse en una conducta diferente e intencional, para orientarse a actividades consideradas significativas" (p.192). De este modo, existe un intento de auto-regulación para la búsqueda y acción hacia metas que incluyan desafío y significado.

El nivel de activación -dimensión fisiológica- que normalmente presenta la persona cuando experimenta aburrimiento, es analizado con más detenimiento a continuación.

Actualmente no hay un acuerdo entre los especialistas sobre el nivel de activación que caracteriza al estado de aburrimiento. Durante mucho tiempo se asumió que la baja activación era su sello característico, pero ahora algunos investigadores afirman que también es posible encontrar aburrimiento con alta activación, o a la vez presentar indicadores de alta y baja activación. Como lo muestra la investigación de "Merrifield y Danckert (2014), quienes encontraron indicaciones de alta y baja activación entre individuos aburridos: (...) el aburrimiento incrementó el ritmo cardíaco y los niveles de cortisol (ambos marcadores de alta activación), pero disminuyó la conductividad de la piel (reflejando baja activación)." (van Tilburg \& Igou, 2017, p.319). Otra posibilidad es la presencia secuencial de ambos niveles de activación, de manera que, primero se presentaría una baja activación, la cual motivaría a la persona realizar acciones para superar el aburrimiento, y a la vez originar una alta activación (Van Tilburg e Igou (2017).

A continuación, se reseñan dos investigaciones vinculadas con el nivel de activación. La primera fue realizado por Acce et al. (2010) utilizando una muestra de estudiantes, quienes frente a situaciones poco exigentes (e.d., baja activación) reaccionaban con aburrimiento, mientras que al experimentar 
como muy exigentes (e.d., alta activación), podían reaccionar según dos modalidades de aburrimiento posibles: percibiendo las tareas como tediosas y no significativas cuando se centraban en las tareas, o con sentimientos de frustración e insatisfacción, al estar centrados en sí mismos.

El otro trabajo pertenece a Harju y Hakanen (2016) quienes realizaron "focus groups" con una muestra de 72 empleados de oficina que laboraban en cuatro empresas de Finlandia, y lograron identificar tres tipos de aburrimiento, uno con baja activación, y los otros con alta activación:1) Inercia.- Sentirse estancado por tener poco trabajo y ejecutar labores rutinarias; 2) Aceleración.- Vivenciar un estado de sobre-activación por alta carga o intenso ritmo laboral, que impiden reflexionar sobre sus acciones o realizar planificaciones futuras; 3) Interrupción del ritmo.- Vivenciar presentes discontinuos, al tener que atender constantes interrupciones en la ejecución de sus labores. Además, los tres tipos difieren según el tiempo vivenciado, pero son iguales al no permitir que la persona utilice plenamente sus capacidades.

Con respecto a los tipos de aburrimiento resaltamos dos por su importancia: situacional o estado y el crónico. El situacional o estado implica una experiencia momentánea de aburrimiento en determinado contexto, pudiendo darse el caso que hasta el mismo estímulo en otras oportunidades no produzca dicha emoción. En el crónico, la persona de manera continua vivencia aburrimiento en un determinado contexto, como podría ser la situación de una secretaria que siempre está aburrida con sus labores cotidianas.

\section{MEDICIÓN DEL ABURRIMIENTO}

$\mathrm{El}$ aburrimiento generalmente es evaluado en base a escalas y cuestionarios. Vodanovich y Watt (2016) identifican 16 escalas existentes en la bibliografía. Incluyen mediciones como rasgo de personalidad (p.ej., propensión al aburrimiento), o para áreas específicas como: el ámbito académico, tiempo libre, la actividad sexual, relaciones interpersonales, etc.

En español se dispone de dos instrumentos: La "Escala de Aburrimiento Laboral (EAL)" elaborado por los psicólogos colombianos Martínez y Rodríguez
-Montalbán (2017). Es unidimensional y consta de 8 ítems.

El segundo instrumento es la "Multidimensional State Boredom Scale" (MSBS) validada al español por Minquez et al. (2015). Incluye 5 sub-escalas: No compromiso, Alta activación, Baja Activación, Desatención, y Percepción del Tiempo. Presenta un total de 29 ítems, y es el único instrumento que posee dos sub-escalas para medir el nivel de activación.

\section{EL ABURRIMIENTO COMO PROCESO}

A continuación, se analizará el aburrimiento desde la perspectiva de su desarrollo a lo largo del tiempo, considerándolo como una emoción diferente a sus causas y consecuencias. Esta distinción es propuesta por van Hooff \& van Hooft (2014), quienes elaboraron una escala para medir aburrimiento a partir de otra existente (Lee,1986), seleccionado aquellos ítems que "...estén basados únicamente (...) en los aspectos afectivos y cognitivos, relacionados al aburrimiento laboral como un estado emocional" (p.352). Los ítems seleccionados fueron: Pienso que mi trabajo es aburrido / Hay largos periodos de aburrimiento en mi trabajo / Mi trabajo avanza muy lentamente/ Generalmente me aburro con mi trabajo / El tiempo parece que avanza lentamente cuando estoy en el trabajo.

Según la Figura 1 se puede observar que el aburrimiento surgiría de la interacción entre las características del medioambiente y las del individuo (Mercer-Lynn, Bar, \& Eastwood, 2014; Fisher, 1991).

Frente a la emoción negativa que vivencia el sujeto, este podría recurrir a determinadas conductas para disminuir o eliminar dicha afectividad y obtener algo más satisfactorio, considerándose así al aburrimiento como una señal para que el sujeto intente un uso más grato de sus recursos (Elpidorou, 2017). Dichas conductas constituyen las Estrategias de Afrontamiento al aburrimiento, que a su vez influirían para obtener resultados positivos o negativos para el sujeto y organización.

A continuación, se indagará con más detalle las dimensiones incluidas en la Figura 1: 
Figura 1. El aburrimiento como proceso

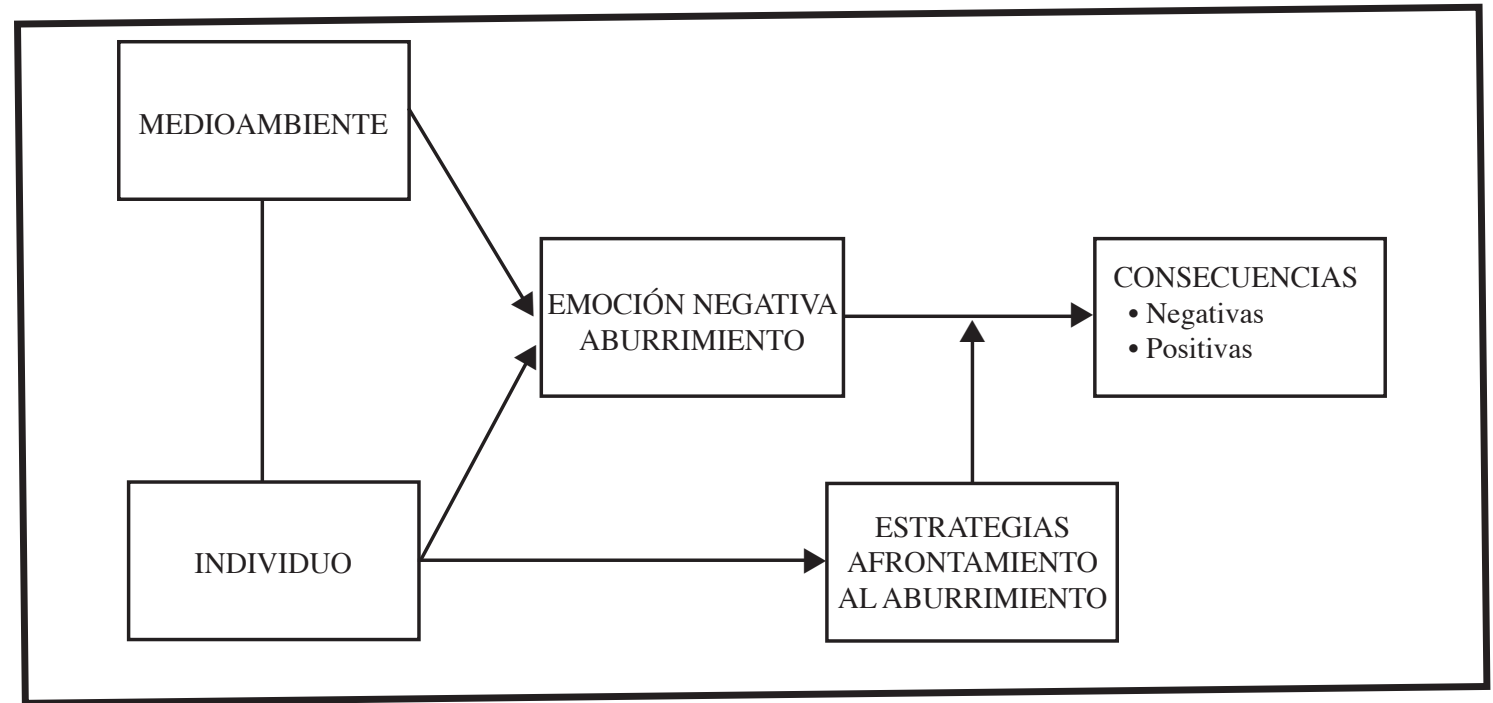

Fuente: Elaborado a partir de Van Hoof \& Van Hoof, 2014

1. El MEDIOAMBIENTE: Destaca las características de los trabajos que desempeñan los empleados. Según las indagaciones empíricas se ha observado que los trabajos rutinarios y poco variados en su contenido tienden a propiciar el aburrimiento (Cummings et al., 2016).

2. CARACTERÍSTICAS DEL INDIVIDUO: Al respecto se ha seleccionado las siguientes dimensiones: Personalidad, Expectativas, Experiencias previas, y satisfacción de las Necesidades Psicológicas Básicas. A continuación, se analiza cada una de ellas.

\subsection{Personalidad}

Algunas personas presentan como rasgo de personalidad la tendencia a vivenciar aburrimiento en las diferentes facetas de su vida, independientemente de las características o cambios del medio externo. Esto se conoce como Propensión al Aburrimiento (Loukidou, Loan-Clarke, \& Daniels, 2009).

Se ha identificado dos tipos de Propensión al Aburrimiento (Struck, Carriere, Cheyne, \& Danckert, 2015a):

- Propensión al Aburrimiento Externo. La persona continuamente está buscando nuevos estímulos externos que le interese, sin lograr permanecer concentrado por mucho tiempo en cada situación.
- Propensión al Aburrimiento Interno. La persona es poco capaz para entretenerse por sí mismo. Carece de la creatividad o imaginación para lograr perspectivas diferentes de una situación o tarea, que le permita mantener su interés.

Desde una perspectiva práctica Struk, Scholer, y Danckert(2015b) sugierenqueatravésdelacapacitación se puede disminuir ambos tipos de Propensión al Aburrimiento. Además, indican que según “...nuestros resultados muestran que la propensión al aburrimiento está fuertemente relacionada a la efectividad de perseguir metas. Dentro de este marco especulamos que la propensión al aburrimiento puede surgir de la incapacidad del sujeto para regularse efectivamente de una manera auto-dirigida." (p.10)

\subsection{Expectativas personales}

Laspersonas generalmente poseen ciertos objetivos que desearían conseguir en un determinado contexto. Por ejemplo, en la situación del estudiante que recién egresa de una universidad, puede anhelar ejercer su profesión disponiendo de ciertas oportunidades, como tener un margen de libertad para decidir, obtener un buen salario, aprender continuamente, etc.

Mael y Jex (2015) consideran que el nivel de congruencia entre las expectativas de las personas y lo que obtienen en realidad, puede ser una posible causa 
para el aburrimiento. Propuesta que también coincide con la aproximación de Schaufeli y Salanova (2014) desde el marco conceptual de "Demandas Laborales" y "Recursos".

Para probar la hipótesis de Mael y Jex (2015), Gkorezis y Kastritsi (2017) utilizaron una muestra de 124 trabajadores griegos pertenecientes a empresas vinculadas a la manufactura, servicios financieros, al cuidado de la salud, y a educación. Demostraron finalmente que el nivel de cumplimiento de las expectativas mostraba una relación positiva con la motivación intrínseca laboral. Además, existía una relación indirecta entre ambas dimensiones, de manera que el cumplimiento de las expectativas se relacionaba negativamente con aburrimiento, y ésta a su vez se vinculaba negativamente con la motivación intrínseca laboral.

\subsection{Experiencias previas}

Mael y Jex (2015) indican la necesidad de considerar ciertas peculiaridades de la actual sociedad para entender mejor las respuestas de aburrimiento de los trabajadores. Según su análisis destacan el uso de la tecnología de la información, la sociedad del entretenimiento y la sobre-estimulación.

Las personas que están acostumbradas a un uso muy frecuente de la tecnología de la información -pudiendo llegar hasta niveles de adicción-, es muy probable que presenten mayores niveles de aburrimiento con sus tareas formales en una organización. Especialmente la generación de los millennials, que están habituados a mantener una comunicación continua con sus pares, manejar información breve y variada, y presentar dificultades para su concentración.

Una característica de las sociedades modernas es la presencia de una multiplicidad de estímulos: luces, letreros, bocinas de autos, música, etc. Frente al cual las personas pueden reaccionar con aburrimiento "... como una función defensiva (...) contra el ruido" (Mael \& Jex, 2015, p. 144). Se agrega a esto, la existencia de una sociedad del entretenimiento o "civilización del espectáculo" (Mario Vargas Llosa, 2012), donde lo principal para las personas es la diversión continua y escapar del aburrimiento. Al respecto, "Makulas (2002) declaró que la pasividad- sentarse y esperar a ser entretenido por el mundo o los demás- es una primera causa del aburrimiento" (Mael \& Jex, 2015, p.145).

\subsection{Satisfacción de las necesidades psicológicas básicas}

La teoría motivacional de la "Auto-determinación" (Gagnè \& Deci, 2005; Van den Broeck, Ferris, Chang, \& Rosen, 2016) ha propuesto, que de manera hereditaria todos los seres humanos necesitan satisfacer tres necesidades básicas para tener un nivel adecuado de bienestar y desarrollo: autonomía para tomar decisiones, tener las competencias necesarias para actuar sobre su entorno, y mantener buenas relaciones con su medio social. A continuación, se reseñan dos investigaciones que incluyen como una variable explicativa la satisfacción de las Necesidades Psicológicas Básicas, dentro de un contexto con presencia de aburrimiento.

Según la investigación realizada por van Hooff y van Hooft (2017) se puede afirmar que, la satisfacción de las Necesidades Psicológicas Básicas en el trabajo y la Motivación Intrínseca Laboral, cumplen un rol mediador entre las características del puesto y el aburrimiento laboral. Específicamente, las características del puesto -y principalmente su variedad, identidad, y autonomía de la tareaspermitían un nivel de satisfacción de las Necesidades Psicológicas Básicas, la cual incidía en la Motivación Intrínseca Laboral, y ésta finalmente en la experiencia de aburrimiento en el trabajo.

Otra conclusión del trabajo de van Hooff y van Hooft (2017), es el efecto del desborde (spillover) de las emociones diarias hacia el próximo día. El nivel de satisfacción o aburrimiento que experimente el trabajador en un determinado día, tiende a predisponer una vivencia similar al día siguiente. Visto esto a largo plazo, nos permite entender mejor cómo se forma una característica más duradera del aburrimiento.

La indagación empírica de van Hooff y van Hooft (2016) logró identificar el rol que pueden cumplir la Centralidad Laboral -cuán importante es el trabajo en la vida del empleado-, y la satisfacción de las Necesidades Psicológicas Básicas en el hogar después de culminar cada jornada laboral. Utilizaron 
una muestra de 106 empleados holandeses en distintas ocupaciones -quienes contestaron vía internet los instrumentos en el transcurso de una semana laboraly concluyeron que la Centralidad Laboral y la satisfacción de las Necesidades Psicológicas Básicas deberían ser considerados para entender la asociación entre el aburrimiento laboral y el bienestar del empleado" (p.209).

Específicamente, según la investigación de van Hooff y van Hooft (2016), existe una relación positiva entre aburrimiento laboral y estado de humor depresivo, sólo para los trabajadores con una alta Centralidad del Trabajo. De manera que, si un empleado otorgaba un rol muy importante a su trabajo, y a la vez experimentaba aburrimiento por las tareas que realizaba, también mostraba reacciones depresivas. La segunda conclusión de este estudio, fue el rol "amortiguador" de la satisfacción de las Necesidades Psicológicas Básicas. Los empleados que conseguían una mayor satisfacción de sus necesidades psicológicas en el hogar, presentaban menores niveles de humor depresivo a pesar de haber experimentado aburrimiento en el trabajo, al compararlo con aquellos con baja satisfacción.

\section{ESTRATEGIAS DE AFRONTAMIENTO AL ABURRIMIENTO}

Hamilton, Haier, y Buschbaum (1984) definieron el Afrontamiento al Aburrimiento (Boredom Coping) como “...una disposición para reestructurar las percepciones y participar en actividades potencialmente aburridas, de manera que disminuya el aburrimiento (y/o maximice las oportunidades para un disfrute intrínseco)" (p. 183). En base a esta definición los autores elaboraron una escala de 10 ítems para la medición del constructo. Posteriormente Game (2007) utilizó dicha escala para elaborar otra, la cual finalmente incluía los siguientes ítems:

- En mi trabajo, generalmente es fácil concentrarme en lo que estoy haciendo

- Usualmente soy capaz de encontrar cierto disfrute en mi trabajo, aún en tareas laborales rutinarias

- Cuando estoy en mi trabajo, el tiempo parece pasar muy lentamente [puntaje invertido]

- Encuentro fácilmente maneras de hacer mi trabajo más interesante
- Cuando estoy aburrido con mi trabajo, es solamente por corto tiempo antes de interesarme de nuevo

- Durante un día promedio de trabajo, generalmente estoy más aburrido que interesado [puntaje invertido]

- En mi trabajo, generalmente puedo encontrar hacer algo que me mantenga interesado.

Game (2007) realizó un estudio utilizando una muestra de 212 trabajadores de una empresa relacionada a productos químicos, a quienes aplicó cuestionarios -incluyendo su escala de Afrontamiento al Aburrimiento- y encontró que los trabajadores con alto nivel en Afrontamiento al Aburrimiento, presentaban un mejor bienestar psicológico y cumplían más los procedimientos de seguridad establecidos por la empresa, al compararlo con aquellos que poseían un bajo nivel.

Skowronski (2012) elaboró una tipología del Afrontamiento al Aburrimiento. Las cuatro categorías propuestas se indican a continuación, incluyendo algunas actividades que pueden expresar los trabajadores:

- Afrontamiento de compromiso conductual: Asumir responsabilidades adicionales / Ayudar a otros empleados / Buscar oportunidades de entrenamiento / Variar la forma de desempeñar sus tareas / Variar la velocidad del desempeño

- Afrontamiento de no compromiso conductual: Socialización no vinculada a las tareas / Promover chismes / Hacer chacota / Excesivo descanso, tardanzas / Abuso de alcohol o sustancias.

- Afrontamiento de compromiso cognitivo: Refocalizandola atenciónen la tarea/Estableciendo metas de desempeño laboral / Redefiniendo la importancia de la tarea / Expandiendo la visión o pensamiento para mejoras.

- Afrontamiento de no compromiso cognitivo: Soñando despierto / Durmiendo/ Reduciendo la concentración en la tarea.

En el ámbito educativo también se ha investigado el Afrontamiento al Aburrimiento del alumno durante las clases (Nett, Goetz, \& Daniels, 2010; Nett, Goetz, \& Hall, 2011; Daniels, Tze, \& Goetz, 2015). 


\section{CONSECUENCIAS}

Dependiendo de las conductas que manifieste la persona se presentarán consecuencias positivas o negativas, tanto para la empresa como al trabajador involucrado. Hasta la fecha se ha otorgado más interés por las consecuencias negativas, pero recientemente se está considerando la influencia positiva del aburrimiento (Elpidorou, 2017a, 2014; Johnsen, 2016).

\subsection{Consecuencias negativas}

Harju, Hakanen, y Schaufeli (2014) realizaron un estudio considerando 87 centros laborales en Finlandia, y concluyeron haber identificado "...una clara asociación entre el aburrimiento laboral y las percepciones negativas relacionadas al trabajo y salud. Estos resultados apoyan la noción que el aburrimiento laboral puede ser dañino para la salud del empleado" (p. 911). En el estudio efectuado por Chin, Markey, Bhargava, Kassam, y Loewenstein (2017) se observó que el aburrimiento es "...predictivo de soledad, cólera, tristeza y preocupación” (p. 359). De manera más explícita, Skowronski (2012) afirma lo siguiente:

Sin considerar sus causas, el aburrimiento laboral es claramente dañino para los individuos y organizaciones. Para los individuos, el aburrimiento relacionado al trabajo a menudo conduce a un incremento en el consumo de alcohol (Wiesner et al., 2005; Ames \& Cunradi, 2004), reducida satisfacción laboral (Game, 2007); MacDonald \& MacIntyre, 1997; Kass et al., 2001), y mayor tensión laboral (Matthews et al., 2000). Además, los empleados aburridos son más probables de estar ausentes (Kass et al., 2001; Dyer-Smith \& Wesson, 1997), retirarse de la organización (Mann, 2007), cumplir menos con los procedimientos de seguridad (Game, 2007), y propensos a accidentes de trabajo (Frone, 1998). Otras consecuencias incluyen, un menor desempeño laboral (Thackray et al., 1977) y conductas laborales contraproducentes más frecuentes (Bruursema, 2007; Spector, et al., 2006). (pp.144-5)

Según la investigación de Moynihan, Igou, y van Tilburg (2017) el aburrimiento incrementa la impulsividad como una respuesta a la poca significatividad que vivencia el sujeto. Dicha influencia es mayor cuando éste presenta como rasgo una elevada auto-conciencia, esto es, presenta interés por analizar sus sentimientos, pensamientos, conocer lo que piensan los demás sobre él, etc.

\subsection{Consecuencias positivas}

Se ha empezado a identificar que en ciertas circunstancias el aburrimiento puede fomentar la creatividad (Mann \& Cadman, 2014), así como facilitar algunos procesos, como la autorregulación, la búsqueda de mejoras para la realización de las tareas, incremento de las intenciones de ayuda social, y promoción de mejores hábitos alimenticios (van Tilburg \& Igou, 2017; Moynihan et al., 2015), e intervenir como un activador para la búsqueda de significado (Coughlan, Igou, van Tilburg, Kinsella, \& Ritchie, 2017).

\section{POSIBLES INVESTIGACIONES SOBRE EL ABURRIMIENTO}

Con la finalidad de lograr un conocimiento más amplio sobre el aburrimiento en las organizaciones, $\mathrm{y}$ especialmente en nuestro contexto donde se ha investigado muy poco, se incluyen supuestas relaciones entre variables, a fin de propiciar el inicio de una indagación más exhaustiva que permita elaborar diseños específicos de investigación. Adicionalmente se proporciona alguna información sobre instrumentos de medición.

Ámbito social. Con énfasis en la relaciones interpersonales:

- Apoyo recibido por los jefes y/o compañeros de trabajo.- Si el trabajador percibe apoyo de parte de su jefe y/o compañeros de trabajo, se esperaría mayor motivación y activación, que su vez facilitarían una disminución del aburrimiento. Medición apoyo social: Benavides et al. (2002).

- Estilosdeliderazgoyaburrimiento--Seesperaría que un estilo de liderazgo transformacional, contribuiría para un menor nivel de aburrimiento en el personal, considerándose que "...muchas investigaciones han revelado que un empleado que recibe apoyo, inspiración y coaching de calidad de parte del supervisor, es probable experimentar el trabajo como más 
desafiante (...)" (Guglielmi, Simbula, Mazzetti, Tabanelli, \& Bonfiglioli, 2013,p.319). Un estilo "servicial" también disminuirá el aburrimiento al fomentar en el personal el auto-rediseño laboral (Harju, Schuafeli, \& Hakanen, 2018). Medidas estilos de liderazgo: Castro, Lupano, Benatuil, y Nader (2007).

Personalidad. Los rasgos de personalidad tienen una influencia en la conducta de los trabajadores:

- Relación entre los rasgos de personalidad y aburrimiento. Según el modelo de personalidad "Big Five", es más probable que las personas con alto nivel de "Conciencia" muestren menor aburrimiento. Medida "Cuestionario Big Five": Caprara, Barbaranelli, \& Borgogni (1995).

Satisfacción y motivación laboral.- Orientado hacia el área afectiva del personal:

- Los trabajadores con mayor satisfacción laboral presentarían menores niveles de aburrimiento

- El personal motivado intrínsecamente, mostraría menos aburrimiento en comparación de aquellos con motivación extrínseca. Medición motivación: Castillo (2014).

- A mayor nivel de Engagament el trabajador presentaría menor aburrimiento (Harju et al., 2016b). Medición Engagement : Schaufeli (2011)

- A mayor Inteligencia Emocional se esperaría menor aburrimiento.

- A mayor satisfacción de las Necesidades Psicológicas Básicas, se esperaría un menor nivel de aburrimiento. Medición satisfacción Necesidades Psicológicas Básicas: Abós, Sevil, Clemente, Martín-Albo, \& García-González (2017).

\section{Relación con otras variables:}

- La Procrastinación en el trabajo estaría relacionada positivamente con el aburrimiento laboral (Vodanovich \& Rupp, 1999; Solf, 2017). Medición Procrastinación Laboral: Guzmán y Rosales (2017).

- Actividades después de las horas de trabajo.-En la investigación sobre el estrés se ha indagado la importancia de la Conducta de Recuperación (Colombo \& Cifre, 2012). Se esperaría menores niveles de aburrimiento en los trabajadores con mayores Conductas de Recuperación. Medida "Cuestionario de Experiencias de Recuperación": Sanz-Vergel et al. (2010).

- A mayor nivel de auto-eficacia menor nivel de aburrimiento. Al ser la auto-eficacia un componente importante de la auto-regulación (Struck et al., 2015b) se asume que estaría relacionada con la respuesta de aburrimiento.

- Características del puesto.- Desempeñar un cargo que permita autonomía en la toma de decisiones, presente variedad de tareas, sus labores son significativas, y provea retroinformación, es más probable que fomente un menor nivel de aburrimiento. De existir incongruencias entre las "características del cargo" y el nivel de instrucción del personal, es factible que se presente más aburrimiento. Medida "características del puesto": Bayona, Caballer, \& Peiró, J-M. (2015).

\section{INTERVENCIONES PARA PREVENIR O DISMINUR EL ABURRIMIENTO}

Las actividades para evitar o disminuir el aburrimiento, deberían orientarse según las causas específicas para cada situación. Para el diagnóstico se debe considerar tarea, trabajador, y contexto medioambiental. Algunas intervenciones posibles al respecto se presentan a continuación:

\section{Adecuación de las tareas}

En situaciones que las tareas sean monótonas o muy rutinarias, puede ser útil el "enriquecimiento laboral" a través del rediseño de las tares para lograr un mayor nivel de activación.

\section{Utilidad de la selección y capacitación}

De tenerse un amplio conocimiento de las características de los puestos, se permitiría identificar las competencias necesarias que deberían poseer los posibles candidatos para un adecuado desempeño. Entre las competencias a evaluar se podría identificar la Predisposición al Aburrimiento, la cual tendría efectos negativos para el desempeño, especialmente en 
cargos con labores rutinarias y que exijan un continuo monitoreo (Cummings et al.,2016).

Desde la perspectiva de la capacitación que normalmente se imparte en los centros de trabajo, se puede intervenir de la siguiente manera:

- Permitir que los trabajadores identifiquen la sintomatología del aburrimiento en su persona y los demás, así como los efectos en el bienestar y desempeño laboral. Indaguen sus habituales "estrategias de afrontamiento al aburrimiento", para reforzar o eliminar determinadas conductas según el caso.

- Fomentar en el trabajador conductas proactivas que permitan modificar el proceso laboral y/o los contenidos de sus tareas, y conseguir así un aporte más funcional. Esto estaría enmarcado dentro del auto-rediseño laboral o "Job Crafting", el cual ha sido demostrado permitir un equilibrio entre demandas y recursos (Harju, Hanaken, \& Schaufeli, 2016).

- Los trabajadores analicen la estrecha interacción entre los contextos trabajo-familia, y se interesen por efectuar los reforzamientos o cambios conductuales según el caso.

- Capacitar a los supervisores sobre todo lo vinculado al aburrimiento y su adecuada gestión en el trabajo.

\section{Roles del supervisor}

Los supervisores de los centros laborales pueden cumplir un rol muy importante para la prevención y manejo eficaz del aburrimiento. Entre sus posibles aportes se puede mencionar lo siguiente:

- Permitir que los subordinados identifiquen de manera clara sus objetivos laborales; proporcionar una adecuada retroinformación de su desempeño; y otorgar recompensas por el buen rendimiento.

- Fomentar en lo posible el trabajo de equipo, lo cual contribuye a “... combatir el aburrimiento al poder proveer a los empleados feedback directo (...) y oportunidades para comprometerse frecuentemente en encuentros sociales coordinados." (Driskell, Driskell, \& Salas, 2014, p.269).

- Proporcionar la suficiente información al trabajador sobre la importancia de sus labores para el logro de las metas organizacionales.

- Realizar un diagnóstico y ejecutar acciones según el nivel de satisfacción de los trabajadores en sus Necesidades Psicológicas Básicas: disponer de un adecuado margen de decisión, poseer las competencias para realizar sus labores, y lograr relaciones sociales positivas (Van den Broeck et al., 2016).

- Ubicar al personal de manera que facilite sus interacciones interpersonales. Al respecto, Zagenczyk,Murrell,yGibney(2007)manifiestan que "...administrando efectivamente el medio ambiente físico laboral, las organizaciones pueden controlar mejor y/o influenciar la frecuencia y naturaleza de las interacciones entre los empleados, lo cual puede resultar en resultados deseables para los empleados y organización." (p.119). Esto coincide con la tendencia actual para implementar "oficinas con espacios abiertos" y eliminar así barreras físicas que impidan el flujo de la comunicación entre los miembros (De Paoli, Arge, \& Blakstad, 2013; Kämpf-Dern \& Konkol, 2017).

- Diseñar y ejecutar programas de rotaciones temporales del personal, entre las diversas áreas o funciones, especialmente con aquellos que presenten altos índices de aburrimiento laboral (Tsai, 2016).

- Fomentar en el personal el interés por lograr un desarrollo de carrera dentro de la organización, de manera que consigan cargos más significativos y desafiantes (Harju, 2017).

- Desarrollar en el personal las habilidades necesarias para una eficaz administración de su tiempo (ver Solf, 2017), la cual permitiría prevenir o disminuir el aburrimiento; vínculo demostrado en el estudio de van der Heijden, Schepers, y Nijssen (2012).

- Realizar actividades que fomenten un clima laboral positivo ("fun at work") como puede ser el reconocimiento de acontecimientos personales (p.ej., cumpleaños), eventos sociales (p.ej., día del padre, madre), reconocimientos de logros profesionales (p.ej., promoción laboral, culminación de estudios). Dicho clima laboral podría incrementar los niveles motivacionales y reforzar las relaciones interpersonales, como lo 
confirman algunas investigaciones al respecto (Fluegge-Woolf, 2014).

\section{COMENTARIOS FINALES}

El presente trabajo ha intentado fundamentar la necesidad de otorgar la debida importancia al aburrimiento que normalmente vivencia el personal, por sus consecuencias generalmente negativas. Dicha emoción no es exclusiva de un tipo de trabajo, al estar presente en la mayoría de las ocupaciones. Desde una perspectiva práctica, es recomendable evitar en lo posible que el aburrimiento se convierta en crónico, por sus consecuencias disfuncionales para el trabajador y organización.

En relación al aspecto metodológico es necesario resaltar las contribuciones de van Hooff y van Hooft (2014), quienes diferenciaron entre emoción y conducta en el ámbito del aburrimiento. Esto ha permitido identificar con mayor precisión causas y consecuencias. Respecto a los instrumentos, sería necesario la creación o adaptación de una escala en español que mida el Afrontamiento al Aburrimiento, y disponer algo similar a las existentes sobre el Afrontamiento del Estrés (Sandín \& Chorot, 2003).

Se requiere mayor investigación sobre la relación entre las dimensiones de la auto-regulación y el aburrimiento (Struk et al.,2015b). Otra área para mayor indagación, y que promete obtener resultados valiosos con fines prácticos y teóricos, es la influencia de las características del ambiente físico laboral sobre determinados aspectos psicológicas del trabajador, incluido el aburrimiento (Zagenczyk, Murrell, \& Gibney, 2007; Hills, \& Levy, 2014; Kallio, Kallio, \& Blomberg, 2015; Harris, 2015; Kämpf-Dern \& Konkol, 2017; Haynes, Suckley, \& Nunnington, 2017).

Finalmente, podemos afirmar que los psicólogos tendrían un rol importante en las organizaciones laborales para el manejo eficaz del aburrimiento, conjuntamente con los demás profesionales que normalmente participan en los centros de trabajo.

\section{REFERENCIAS}

Abós, A., Sevil, J., Clemente, J.A.J., Martín-Albo, \& García-González, L. (2017). Spanish validation of the Basic Psychological Needs at Work Scale. A measure to predict teachers' well-being in the workplace. International Journal for Educational and Vocational Guidance, DOI 10.1007/s10775017-9351-4

Ace, T.W., Kim, H., Kim, H. J., Kim, J-In., Chu, H-N R., Kim. M., \& Wicker, F.W. (2010). Academic in under- and over-challenging situations. Contemporary Educational Psychology, 35, 1727

Balzer, W.K., Jex, S.M., \& Geimer, J. L. (2017). Boredom. Reference Module in Neuroscience and Biobehavioral Psychology, http://dx.doi. org/10.1016/B978-0-12-809324-5.05487-0

Bayona, J.A., Caballer, A., \& Peiró, J-M. (2015). The Work Design Questionnaire: Spanish version and validation. Journal of Work and Organizational Psychology, 31, 187-200. Recuperado de:http:// www.sciencedirect.com/science/article/pii/ S1576596215000389 [6 Agosto 2016]

Benavides, F.G., Gimeno, D., Benach, J., Martínez, J.M., Jarque, S., Berra, A. \& Devesa, J. (2002). Descripción de los factores de riesgo psicosocial en cuatro empresas. Gaceta Sanitaria, 16(3), 222229

Caprara, G.V., Barbaranelli, C., \& Borgogni, L. (1995). BFQ. Cuestionario “Big Five”. Madrid: TEA

Castillo, M.F. (2014). Clima, Motivación Intrínseca y Satisfacción Laboral en trabajadores de diferentes niveles jerárquicos, Tesis Licenciatura Psicología no publicada, PUCP, Lima. Recuperado de: http://tesis.pucp.edu.pe/repositorio/ handle/123456789/5431 [Agosto 2016]

Castro, A., Lupano, M.L., Benatuil, D., \& Nader, M. (2007). Teoría y Evaluación del Liderazgo, Buenos Aires: Paidós

Chin, A., Markey, A., Bhargava, S., Kassam, K.S., \& Loewenstein, G. (2017). Bored in the USA: Experience sampling and boredom in everyday life. Emotion, 17(2), 359-368

Colombo, V., \& Cifre, E. (2012). La importancia de recuperarse del trabajo: Una revisión del dónde, 
cómo y por qué. Papeles del Psicólogo, 33(2), 129-137

Coughlan, G., Igou, E.R., van Tilburg, W.A.P., Kinsella, E.L., \& Ritchie, T.D. (2017). On boredom and perceptions of heroes: A meaning-regulation approach to heroism. Journal of Humanistic Psychology, 1-19DOI: 10.1177/002216817705281

Cummings, M.L., Gao, F., \& Thornburg, K.M. (2016). Boredom in the workplace: A new look at an old problem. Human Factors, 58(2), 279-300

Daniels, L.M., Tze, V.M.C., \& Goetz, T. (2015). Examining boredom: Different causes for different coping profiles. Learning and Individual Differences, 37, 255-261

De Paoli, D., Arge, K, \& Blakstand, S.H. (2013). Creating business value with open space flexible offices. Journal of Corporate Real Estate, 3/4, 181-193

Driskell, T., Driskell, J.E., \& Salas, E. (2014). Stress, Performance, and Decision Making in Organizations. En S. Highhouse, R.S.Dalal, \& E.Salas (Eds.). Judgment and Decision Making at Work (pp. 251-276), New York, NY: Routledge

Eastwood, J.D., Frichen, A., Fenske, M.J., \& Smilek, D. (2012). The unengaged mind: Defining boredom in terms of attention. Perspectives on Psychological Science, 7(5), 482-495

Elpidorou, A. (2014). The bright side of boredom. Frontiers in Psychology, DOI: 10.3389/ fpsyg.2014.01245

Elpidorou, A. (2017a). The good of boredom. Philosophical Psychology, DOI:10.1080/095150 89.2017.1346240

Elpidorou, A. (2017b). The bored mind is a guiding mind: Toward a regulatory theory of boredom. Phenomenology and the Cognitive Sciences, https://doi.org/10.1007/s11097-017-9515-1

Fisher, C. D. (1991). Boredom at work: a neglected concept. School of Business Discussion Papers. Paper 19. http://epublications.bond.edu.au/ discussion_papers/19

Fluegge-Woolf, E.R. (2014). Play hard, work hard: Fun at work and job performance. Management Research Review, 37(8), 682-705
Gagnè, M., \& Deci, E.L. (2005). Self-determination theory and work motivation. Journal of Organizational Behavior, 26, 331-362

Game, A.M. (2007). Workplace boredom coping: Health, safety, and HR implications. Personnel Review, 36(5), 701-721

Guglielmi, D., Simbula, S., Mazzetti, G., Tabanelli, \& Bonfiglioli, R. (2013). When the job is boring: The role of boredom in organizational contexts. Work, 45, 311-322

Guzmán, M.A., \& Rosales, C.I. (2017). Validación de la Escala de Procrastinación en el Trabajo (PAWS) al habla hispana, Tesis Licenciatura Psicología no publicada, Universidad San Ignacio de Loyola, Lima. Recuperado: http://repositorio.usil.edu.pe/ bitstream/USIL/2765/1/2017_Guzm\%C3\%A1n_ Validacion-de-la-escala-de-procrastinacion.pdf [15 Setiembre 2017]

Gkorezis. P., \& Kastritsi, A. (2017). Employee expectations and intrinsic motivation: Workrelated boredom as a mediator, Employee Relations, 39 (1), 100-111

Hamilton, J.A., Haier, R.J., \& Buchsbaum, M. S. (1984). Intrinsic enjoyment and boredom coping scales with personality, evoked potential and attention measures. Personality and Individual Differences, 5(2), 183-193

Harju, L.K., Hakanen, J.J., \& Schaufeli, W.B. (2014). Job boredom and its correlates in 87 Finnish organizations. JOEM, 56(9), 911-918

Harju,L.K., \& Hakanen, J.J. (2016a). An employee who was not there: a study of job boredom in whitecollar work. Personnel Review, 45(2), 374-391

Harju, L.K., Hakanen, J.J, \& Schaufeli, W.B. (2016b). Can job crafting reduce job boredom and increase work engagement? A three-year cross-lagged panel study. Journal of Vocational Behavior, 9597, 11-20

Harju, L.K. (2017). Taming the beast of boredom: Exploring boredom at work and the role of Job Crafting. Tesis Ph.D. no publicado, Aalto University, Recuperado de: https://aaltodoc. aalto.fi/handle/123456789/27337 [6 Nov. 2017]

Harju,L.K., Schaufeli, W.B., \& Hakanen, J.J. (2018). A multilevel study on servant leadership, job 
boredom and job crafting. Journal of Managerial Psychology. $\quad$ https://doi.org/10.1108/JMP-082016-0237

Harris, R. (2015). The changing nature of the workplace and the future of office space. Journal of Property Investment \& Finance, 33(5), 424-435

Haynes, B., Suckley, L., \& Nunnington, N. (2017). Workplace productivity and office type. Journal of Corporate Real Estate, 19(2), 111-138

Hills, R., \& Levy, D. (2014). Workplace design and fit-out: What knowledge workers value. Property Management, 32(5), 415-432

Johnsen, R. (2016). Boredom and organization studies. Organization Studies, 37(10), 1-13. DOI: $10.1177 / 0170840616640849$

Kallio, T.J., Kallio, K-M., \& Blomberg, A.J. (2015). Physical space, culture and organizational creativity- a longitudinal study. Facilities, 33(5/6), 389-411

Kämpf-Dern, A., \& Konkol, J. (2017). Performanceoriented office environments-framework for effective workspace design and the accompanying change processes. Journal of Corporate Real Estate, 19(4), 208-238

Lee, T.W. (1986). Toward the development and validation of a measure of Job Boredom. Manhattan College Journal of Business, 15(1), 22-28

Loukidou, L., Loan-Clarke,J., \& Daniels, K. (2009). Boredom in the workplace: More than monotonous tasks. International Journal of Management Review, 11(4), 381-405

Mael, F., \& Jex, S. (2015). Workplace Boredom: An integrative model of traditional and contemporary approaches. Group \& Organization Management, 10(2), 131-159

Mann, S., \& Cadman, R. (2014). Does being bored make us more creative? Creativity Research Journal, 26(2), 165-172

Martínez, M., \& Rodríguez-Montalbán, R. (2017). Cuando el trabajo aburre: Análisis de las propiedades psicométricas de la Escala de Aburrimiento Laboral (EAL). Revista Interamericana de Psicología Ocupacional, 35(1), 7-20. Doi: 10.21772/ripo.v35n1a01
Mercer-Lynn, K.B., Bar, R.J., \& Eastwood, J.D. (2014). Cause of boredom: The person, the situation, or both? Personality and Individual Differences, 56, 122-126

Minquez, M.A., Montero-Marín, J., Gili, M., PueblaGuedea, M., Herrera-Mercadal, P., Navarro-Gil, M., \& García-Campayo, J. (2015). Validation of Spanish version of the Multidimensional State Boredom Scale (MSBS).Health and Quality ofLife Outcomes, 13:59. Recuperado de:https://pdfs.semanticscholar.org/ e39c/6e3ccf05232e3d1548a962fab366e11ac90f. pdf [4 Abril 2016]

Moynihan, A.B., van Tilburg, W.A.P., Igou, E.R., Wisman, A., Donnelly, A.E., \& Mulcaire, J.B. (2015). Eaten up by boredom: Consuming food to escape awareness of the bored self. Frontiers in Psychology, 6,1-10

Moynihan, A.B., Igou, E.R., \& van Tilburg, W.A.P. (2017).Boredom increases impulsiveness: A meaning-regulation perspective. Social Psychology, 48(5), 293-309

Nett, U.E., Goetz, T., \& Daniels, L.M. (2010). What to do when feeling bored? Students's strategies for coping with boredom. Learning and Individual Differences, 20(6), 626-638

Nett, U.E., Goetz, T., \& Hall, N.C. (2011). Coping with boredom in school: An experience sampling perspective. Contemporary Educational Psychology, 36(1), 49-59

Sandín, B., \& Chorot, P. (2003). Cuestionario de afrontamiento del estrés (CAE): Desarrollo y validación preliminar. Revista de Psicopatología y Psicología Clínica, 8(1), 39-54

Sanz-Vergel, A.I., Sebastián, J., Rodríguez-Muñoz, A., Garrosa, E., Moreno-Jiménez, B., \& Sonnentag, S. (2010). Adaptación del "Cuestionario de Experiencias de Recuperación" a una muestra española. Psicothema, 22 (4), 990-996

Schaufeli, W.B. (2011). Utrech Work Engagement, Recuperado de: http://www.wilmarschaufeli.nl/ publications/Schaufeli/Test\%20Manuals/Test manual_UWES_Espanol.pdf [Diciembre $18 \mathrm{del}$ 2017]

Schaufeli, W.B., \& Salanova, M. (2014). Burnout, Boredom and Engagement in the Workplace. En 
M.C.W. Peeters., J. de Jonge, J., \& T. Taris (Eds.). An introduction to contemporary work psychology (pp. 293-320). Malden, MA: Wiley Blackwell

Skowronski, M. (2012). When the bored behave badly (or exceptionally). Personnel Review, 41(2), 143159

Solf,A.(2017).Tiempo productivoenlasorganizaciones modernas: Aportes de la Psicología. Avances en Psicología, Unifé, 25(1), 9-26

Struck, A.A., Carriere, J.S.A., Cheyne, J.A., \& Danckert, J. (2015a). A Short Boredom Proponeness Scale: Development and psychometric properties. Assessment, 1-14. DOI: 10.1177/1073191115609996

Struk, A.A., Scholer, A.A., \& Danckert, J. (2015b). A self-regulatory approach to understanding boredom proneness. Cognition and Emotion. http://dx.doi.org/10.1080/02699931.2015.10643 63

Tsai, C-J. (2016). Boredom at work and job monotony: An exploratory case study within the catering sector. Human Resource Development Quarterly, 27(2), 207-236

Vargas, M. (2012). La civilización del Espectáculo. Madrid: Alfaguara

Van den Broeck, A., Ferris, D.L., Chang, C-H., \& Rosen,C.C. (2016). A review of self-determination theory's Basic Psychological Needs at work. Journal of Management, 42(5), 1195-1229

Van der Heijden, G.A.H., Schepers, J.J.L., \& Nijssen, E.J. (2012). Understanding workplace boredom among white collar employees: Temporary reaction and individual differences. European Journal of Work and Organizational Psychology, 21(3), 349-375

VandeWalle (1997). Development and validation of a work domain Goal Orientation instrument.
Educational and Psychological Measurement, 57(6), 995-1015

Van Hooff, M.L.M., \& van Hooft, E.A.J. (2014). Boredom at work: Proximal and distal consequences of affective work-relates boredom. Journal of Occupational Health Psychology, 19(3), 348-359

Van Hooff, M.L.M., \& van Hooft, E.A.J. (2016). Work-related boredom and depressed mood from a daily perspective: The moderating roles of work centrality and need satisfaction. Work \& Stress, 30(3), 209-277

Van Hooff, M.L.M., \& van Hooft, E.A.J. (2017). Boredom at work: Towards a dynamic spillover model of need satisfaction, work motivation, and work-related boredom. European Journal of Work and Organizational Psychology, 26(1), 133-149

Van Tilburg, W.A.P., \& Igou, E.R. (2012). On boredom: Lack of challenge and meaning as distinct boredom experiences. Motivation and Emotion, $33,181-194$

Van Tilburg, W.A.P., \& Igou, E.R. (2017). Boredom beg to differ: Differentiation from other emotions. Emotions, 17(2), 309-322

Vodanovich, S. J., \& Rupp, D.E. (1999). Are procrastinators prone to boredom? Social Behavior and Personality, 27(1), 11-16

Vodanovich, S.J., \& Watt, J.D. (2016). Self-Report Measures of Boredom: An Updated Review of the Literature. The Journal of Psychology, 150(2), 196-228

Zagenczyk, T.J., Murrell, A.J., \& Gibney, R. (2007). Effects of the physical work environment on the creation of individual- and group-level social capital. International Journal of Organizational Analysis. 15(2), 119-135

Fecha de recepción: 8 de marzo, 2018

Fecha de aceptación: 17 de junio, 2018 